\title{
论 文
}

\section{测量诱发的关联分布}

\author{
席政军*，李永明 \\ 陕西师范大学计算机科学学院, 西安 710062 \\ *联系人, E-mail: xizhengjun@snnu.edu.cn \\ 收稿日期: 2012-11-01; 接受日期: 2013-01-30 \\ 国家自然科学基金 (批准号: 11271237, 61228305) 和中央高校基本科研业务费专项基金 (编号: GK201302054) 资助
}

摘要量子测量诱发量子系统的扰动使得它与辅助系统以及测量装置之间的关联发生了变化. 本文使用摘 交换与量子失协, 在间接测量模型中, 讨论测量诱发的关联分布, 并得到全关联和量子关联的平衡关系. 最后 讨论局部操作导致量子失协的变化与测量诱发的关联分布之间的关系.

关键词量子测量, 熵交换, 量子关联, 熵增量

PACS: 03.67.-a, 03.67.Pp, 03.65.Yz

doi: $10.1360 / 132012-903$

在量子力学中, 量子测量不可避免地引起量子 系统的扰动 $[1,2]$. 量子测量一般由一个完全正定的保 迹的 (Completely Positive and Trace Preserving, CPTP) 映射来描述. 根据文献 [1], 量子测量可以建模为一 个间接测量模型 (Indirect Measurement Model), 该模 型由辅助量子系统, 被测量子系统以及测量装置构 成. 从信息论的角度出发, 对于该模型已有一些相关 的讨论见文献 [3-7]. 在间接测量模型中, 测量导致 被测量子系统与测量装置之间关联产生, 该关联被 看作是测量诱发的关联, 包括量子关联和经典关联. 同时测量也必然导致被测量子系统与辅助系统之间 关联的变化. 一个自然的问题是: 测量诱发的被测 量子系统和测量装置之间的关联与它和辅助系统之 间的关联变化之间是否存在关系, 甚至存在某种守 恒的关系? 已有研究表明: 量子测量导致它与辅助系 统之间的纠缠会减少 ${ }^{[8]}$. 但是不清楚纠缠的减少与 被测量子系统和测量装置间的关联产生之间的关系,
相关的讨论见文献 [3-6].

在本文中, 利用熵交换 ${ }^{[8]}$ 、Holevo 信息以及量 子失协 ${ }^{[9-11]}$, 我们详细讨论间接测量模型中关联的 分布, 并得到一些重要的守恒关系. 在间接测量模型 中, 我们解释局域操作导致量子失协的变化与测量 诱发的关联分布之间的关系, 以及与局部操作产生 的 Holevo 信息的关系.

\section{1 间接测量模型}

为刻画测量诱发的关联变化, 首先介绍间接测 量模型. 不妨设测量算子由 $\left\{K_{i}^{\dagger} K_{i}\right\}$ 来描述, 根据量 子测量假设, 对于量子系统 $Q$ 的初始量子态 $\rho^{Q}$, 结 果 $i$ 出现的概率为 $p_{i}=\operatorname{Tr}\left(K_{i}^{\dagger} K_{i} \rho^{Q}\right)$, 同时测量后的量 子态为 $\tilde{\rho}_{i}^{Q}=\frac{K_{i} Q K_{i}^{\dagger}}{\operatorname{Tr}\left(K_{i}^{\dagger} K_{i} \rho\right)}$. 从而测量获得系统 $Q$ 上的一 个混合态系综 $\left\{p_{i}, \tilde{\rho}_{i}^{Q}\right\}$, 此时系统 $Q$ 上测量后的态为

$$
\Phi\left(\rho^{Q}\right)=\sum_{i} p_{i} \tilde{\rho}_{i}^{Q} .
$$


该测量也可以称作量子运算或者量子信道 ${ }^{[12]}$. 该测 量过程可以建模为一个三体量子系统上的西变换, 见 图 1. 假设被测量子系统 $\mathscr{H}_{Q}$ 与辅助量子系统 $\mathscr{H}_{R}$ 初 始处于纯态 $|\Psi\rangle^{R Q}$, 且满足 $\rho^{Q}=\operatorname{Tr}_{R}\left(|\Psi\rangle^{R Q}\langle\Psi|\right)$. 测量 装置也是一个量子系统, 记为 $\mathscr{H}_{E}$, 初始态启动于纯 态 $|0\rangle^{E}$, 由此辅助系统 - 被测系统 - 测量装置构成了 三体量子系统, 简记为 $R Q E$. 该三体系统的初始态为 纯态 $|\Psi\rangle^{R Q E}=|\Psi\rangle^{R Q}|0\rangle^{E}$, 在系统 $Q$ 上的量子测量 $\Phi$ 过程等价于系统 $R Q E$ 上的西演化, 用 $|\tilde{\Psi}\rangle^{R Q E}$ 来标记 演化后的态, 即

$$
|\tilde{\Psi}\rangle^{R Q E}=\left(I d^{R} \otimes U^{Q E}\right)|\Psi\rangle^{R Q E} .
$$

事实上, 对于系统 $R Q$ 和 $Q E$ 的演化也可直接表示为

$$
\begin{gathered}
\tilde{\rho}^{R Q}=(I d \otimes \Phi)\left(|\Psi\rangle^{R Q}\langle\Psi|\right), \\
\tilde{\rho}^{Q E}=U\left(\rho^{Q} \otimes|0\rangle^{E}\langle 0|\right) U^{\dagger}=\sum_{i, j} K_{i} \rho^{Q} K_{j}^{\dagger} \otimes|i\rangle\langle j|,
\end{gathered}
$$

其中 $\{|i\rangle\}$ 是测量装置的一组标准正交基. 在测量装 置上取偏迹可得被测量子系统 $\mathscr{H}_{Q}$ 在测量后的量子 态 (仅考虑统计结果), 即

$$
\tilde{\rho}^{Q}=\Phi\left(\rho^{Q}\right)=\operatorname{Tr}_{E}\left[U\left(\rho^{Q} \otimes|0\rangle^{E}\langle 0|\right) U^{\dagger}\right]=\sum_{i} K_{i} \rho^{Q} K_{i}^{\dagger},
$$

其中 $K_{i}$ 是 Kraus 算子, 满足 $\sum_{i} K_{i}^{\dagger} K_{i}=I^{Q}$. 量子运算 $\Phi$ 的补运算 $\hat{\Phi}$ 定义为

$\hat{\Phi}\left(\rho^{Q}\right)=\operatorname{Tr}_{Q}\left[U\left(\rho^{Q} \otimes|0\rangle^{E}\langle 0|\right) U^{\dagger}\right]=\sum_{i, j} \operatorname{Tr}\left(K_{j}^{\dagger} K_{i} \rho^{Q}\right)|i\rangle\langle j|$.

该运算描述测量装置上的量子态, 即 $\tilde{\rho}^{E}=\hat{\Phi}\left(\rho^{Q}\right)$.

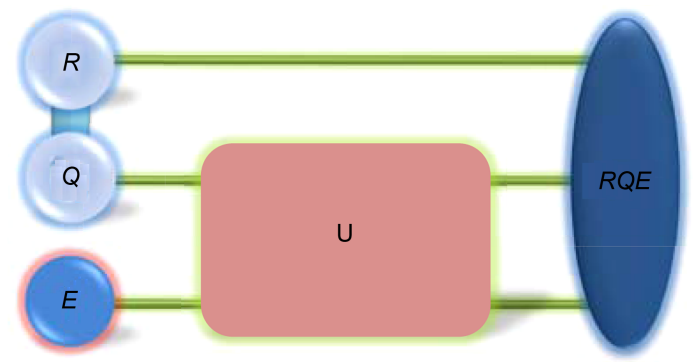

图 1 (网络版彩图) 间接测量模型

被测量子系统 $Q$, 辅助系统 $R$ 以及测量装置 $E$

Figure 1 (Color online) The indirect measurement model. The measured system $Q$, the auxiliary system $R$ and the apparatus $E$.
上述描述过程就是间接测量的基本模型，下面 将讨论在此模型中, Holevo 信息、熵交换以及熵增量 之间的基本关系. 量子测量 (量子运算) $\Phi$ 在量子系统 $Q$ 的状态 $\rho^{Q}$ 上的熵交换 ${ }^{[12]}$ 定义为

$$
S\left(\rho^{Q}, \Phi\right):=S\left(\tilde{\rho}^{R Q}\right) .
$$

由于三体系统 $R Q E$ 在测量前后始终是纯态, 摘交换 等于测量后系统 $E$ 的熵, 即 $S\left(\tilde{\rho}^{R Q}\right)=S\left(\hat{\Phi}\left(\rho^{Q}\right)\right)$.

测量后系统 $Q$ 上的态由系综 $\left\{p_{i}, \tilde{\rho}_{i}^{Q}\right\}$ 来描述, 该 系综的 Holevo 信息 ${ }^{[12,13]}$ 定义为

$$
\chi\left(\left\{p_{i}, \tilde{\rho}_{i}^{Q}\right\}\right):=S\left(\sum_{i} p_{i} \tilde{\rho}_{i}^{Q}\right)-\sum_{i} p_{i} S\left(\tilde{\rho}_{i}^{Q}\right) .
$$

显然, Holevo 信息不会超过系统 $Q$ 测量后的熵, 即

$$
\chi\left(\left\{p_{i}, \tilde{\rho}_{i}^{Q}\right\}\right) \leqslant S\left(\Phi\left(\rho^{Q}\right)\right) .
$$

最近, 文献 [5] 给出 Holevo 信息不会超过熵交换, 即

$$
\chi\left(\left\{p_{i}, \tilde{\rho}_{i}^{Q}\right\}\right) \leqslant S\left(\tilde{\Phi}\left(\rho^{Q}\right)\right) .
$$

再使用不等式 $\sum_{i} p_{i} S\left(\tilde{\rho}_{i}^{Q}\right) \leqslant S\left(\rho^{Q}\right)$, 简单运算得到 Holevo 信息的上界和下界, 即

$$
\Delta S_{Q} \leqslant \chi\left(\left\{p_{i}, \tilde{\rho}_{i}^{Q}\right\}\right) \leqslant \Delta S_{E},
$$

其中 $\Delta S_{Q}=S\left(\Phi\left(\rho^{Q}\right)\right)-S\left(\rho^{Q}\right)$ 是测量导致的系统 $Q$ 的熵增量. 由于测量装置 $E$ 的初始态为纯态, 故而 $S\left(\hat{\Phi}\left(\rho^{Q}\right)\right)$ 看作是测量导致的系统 $E$ 上的熵增量, 记 作 $\Delta S_{E}=S\left(\hat{\Phi}\left(\rho^{Q}\right)\right)$.

该结果说明: 被测系统上获得的 Holevo 信息不 小于测量导致的熵增量, 且不超过测量装置上的熵增 量. 一般情况下, 量子测量导致的熵增量有可能是负 的, 而且也很难直接比较 $S\left(\Phi\left(\rho^{Q}\right)\right)$ 和 $S\left(\hat{\Phi}\left(\rho^{Q}\right)\right)$, 综 上讨论我们给出 Holevo 信息的一个紧凑的上下界, 即

$$
\begin{aligned}
\max \left\{0, \Delta S_{Q}\right\} & \leqslant \chi\left(\left\{p_{i}, \tilde{\rho}_{i}^{Q}\right\}\right) \\
& \leqslant \min \left\{S\left(\Phi\left(\rho^{Q}\right)\right), S\left(\tilde{\Phi}\left(\rho^{Q}\right)\right)\right\} .
\end{aligned}
$$

该等式说明若系统 $Q$ 的熵增量越大, 获得的 Holevo 信息越大, 同时熵交换就越大. 结合 Fano 不 等式, 这蕴含着系统 $Q$ 的熵增量越大, 系统 $R Q$ 之间 
的纠缠就越不能得到好的保持. 同时测量装置 $E$ 的 熵增量越大.

此时有两个基本的问题产生: 系统 $R Q$ 之间的纠 缠减少与系统 $E Q$ 之间纠缠产生是否存在关系? 已 有研究表明即使是可分混合态也具有量子关联. 由 于系统 $Q$ 上的测量使得系统 $R Q$ 变为混合系统, 那 么系统 $R Q$ 之间的关联变化与系统 $E Q$ 之间关联产 生是否存在联系?

\section{2 关联分布}

从上一节可知, 在系统 $Q$ 上的测量导致系统 $R Q$ 的纠缠减少以及系统 $Q E$ 的关联的增加. 这节重点 讨论系统 $Q$ 上的测量导致系统 $R Q$ 的关联减少与系 统 $Q E$ 的关联增加之间的关系. 不失一般性, 对于两 体系统, 使用量子互信息去度量全部关联, 使用量子 失协来度量量子关联.

为了讨论测量诱发的关联分布, 首先给出量子 失协的定义. 在系统 $Q$ 上测量后, 系统 $R Q$ 上的量子 态为 $\tilde{\rho}^{R Q}$, 为给出量子失协 (量子关联), 考虑在系统 $Q$ 上再次进行测量, 则得到关于辅助系统 $R$ 的最大 Holevo 信息被定义为经典关联 ${ }^{[9]}$, 即

$$
\mathscr{C}\left(\rho^{R Q}\right):=S\left(\tilde{\rho}^{R}\right)-\min _{\left\{\Pi_{k}^{Q}\right\}} \sum_{k} p_{k} S\left(\tilde{\rho}_{k}^{R}\right),
$$

其中 $\left\{\Pi_{k}^{Q}\right\}$ 是在子系统 $Q$ 上的 rank-1 的 POVM, 在测 量后得到结果为 $k$ 的概率为 $p_{k}=\operatorname{Tr}\left(I^{R} \otimes \Pi_{k}^{Q} \tilde{\rho}^{R Q}\right)$, 且 对应的子系统 $R$ 上的态为 $\tilde{\rho}_{k}^{R}=\operatorname{Tr}_{Q}\left(I^{R} \otimes \Pi_{k}^{Q}\right) / p_{k}$. 量 子关联定义为全关联和经典关联的差 ${ }^{[10]}$, 即

$$
\delta(R \mid Q):=\mathscr{I}\left(\rho^{R Q}\right)-\mathscr{C}\left(\rho^{R Q}\right),
$$

其中 $\mathscr{I}\left(\rho^{R Q}\right)=S\left(\tilde{\rho}^{R}\right)+S\left(\tilde{\rho}^{Q}\right)-S\left(\tilde{\rho}^{R Q}\right)$ 是量子态 $\tilde{\rho}^{R Q}$ 的互信息, 可看作是全部关联度量 ${ }^{[10,11]}$.

\section{1 全关联的守恒}

在系统 $Q$ 被测量前后, 系统 $R Q$ 之间的全关联分 别为

$$
\begin{aligned}
& \mathscr{I}_{\mathrm{b}}(R: Q)=2 S\left(\rho^{Q}\right), \\
& \mathscr{I}_{\mathrm{f}}(R: Q)=S\left(\tilde{\rho}^{R}\right)+S\left(\tilde{\rho}^{Q}\right)-S\left(\tilde{\rho}^{R Q}\right) .
\end{aligned}
$$

这里的下角标 $\mathrm{b}, \mathrm{f}$ 表示测量前后. 从而系统 $R Q$ 在演 化过程中全关联的增量可表示为

$$
\begin{aligned}
\Delta_{\mathscr{I}(R: Q)} & =\mathscr{I}_{\mathrm{f}}(R: Q)-\mathscr{I}_{\mathrm{b}}(R: Q) \\
& =S\left(\tilde{\rho}^{Q}\right)-S\left(\tilde{\rho}^{R Q}\right)-S\left(\rho^{Q}\right) .
\end{aligned}
$$

同理系统 $Q$ 被测量前后, 系统 $Q E$ 之间的全关联分 别为

$$
\begin{aligned}
& \mathscr{I}_{\mathrm{b}}(Q: E)=0, \\
& \mathscr{I}_{\mathrm{f}}(Q: E)=S\left(\tilde{\rho}^{Q}\right)+S\left(\tilde{\rho}^{E}\right)-S\left(\tilde{\rho}^{Q E}\right) .
\end{aligned}
$$

系统 $Q E$ 在演化过程中全关联的增量为

$$
\begin{aligned}
\Delta_{\mathscr{I}(Q: E)} & =\mathscr{I}_{\mathrm{f}}(Q: E)-\mathscr{I}_{\mathrm{b}}(Q: E) \\
& =S\left(\tilde{\rho}^{Q}\right)+S\left(\tilde{\rho}^{E}\right)-S\left(\rho^{Q}\right) .
\end{aligned}
$$

结合等式 (16) 和 (17), 得到下面等式

$$
\Delta_{\mathscr{I}(Q: E)}-\Delta_{\mathscr{I}(R: Q)}=2 S\left(\rho^{Q}, \Phi\right) .
$$

该等式说明: 系统 $Q$ 上的测量导致系统 $R Q$ 的全关 联的增量与系统 $Q E$ 的全关联增量的差等于系统 $Q$ 上的熵交换的 2 倍.

\section{2 量子关联的守恒}

考虑演化后的三体纯态系统 $R Q E$, 基于在系统 $Q$ 上测量, 使用 Koashi-Winter 关系 ${ }^{[15]}$ 以及文献 [16] 的结果, 系统 $R Q$ 与 $Q E$ 之间的量子关联分别为

$$
\begin{aligned}
& \delta(R \mid Q)=S\left(\tilde{\rho}^{Q}\right)-S\left(\tilde{\rho}^{R Q}\right)+E\left(\tilde{\rho}^{R E}\right), \\
& \delta(E \mid Q)=S\left(\tilde{\rho}^{Q}\right)-S\left(\tilde{\rho}^{Q E}\right)+E\left(\tilde{\rho}^{R E}\right) .
\end{aligned}
$$

这里 $E\left(\tilde{\rho}^{R E}\right)$ 是系统 $R E$ 在演化后的形成纠缠熵. 为 了讨论系统 $R Q$ 的量子关联的减少程度与系统 $Q E$ 的量子关联的增加的关系, 将上两式相减得到

$$
\delta(R \mid Q)-\delta(E \mid Q)=S\left(\tilde{\rho}^{Q E}\right)-S\left(\tilde{\rho}^{R Q}\right) .
$$

由于系统 $R$ 不涉及 $Q$ 和 $E$ 发生西相互作用, 因此有

$$
S\left(\tilde{\rho}^{Q E}\right)=S\left(\rho^{Q}\right) .
$$

再利用第一次测量后系统 $R Q E$ 的纯性可知

$$
\delta(R \mid Q)-\delta(E \mid Q)=S\left(\rho^{Q}\right)-S\left(\rho^{Q}, \Phi\right) .
$$


在第一次测量前系统 $R Q$ 是纯化态, 它的量子关联等 于约化态的熵. 若记 $\Delta_{\delta(R \mid Q)}=\delta(R \mid Q)-S\left(\rho^{Q}\right)$ 表示系 统 $R Q$ 由测量导致的量子关联的增量. 由于系统 $Q E$ 在西作用前是直积态, 从而 $\delta(E \mid Q)$ 表示系统 $Q E$ 在 西作用下量子关联的增量, 记作 $\Delta_{\delta(E \mid Q)}=\delta(E \mid Q)$. 进 而得到测量诱发的量子关联分布的守恒关系, 即

$$
\Delta_{\delta(E \mid Q)}-\Delta_{\delta(R \mid Q)}=S\left(\rho^{Q}, \Phi\right) .
$$

该等式说明: 系统 $Q$ 上的测量诱发的系统 $R Q$ 的量子 关联的增量与系统 $Q E$ 的量子关联的增量的差等于 熵交换. 换句话说, 系统 $Q$ 上的测量诱发的系统 $R Q$ 的量子关联的减少与系统 $Q E$ 的量子关联增加的和 等于熵交换. 该等式也说明测量诱发的量子关联的 分布是全关联分布的一半.

结合式 (23) 和式 (24), 得到测量获得的系统 $Q$ 上的 Holevo 信息不超过测量诱发的系统 $R Q$ 的量子 关联的增量与系统 $Q E$ 的量子关联的增量的差, 即

$$
\chi\left(\left\{p_{i}, \tilde{\rho}_{i}^{Q}\right\}\right) \leqslant \Delta_{\delta(E \mid Q)}-\Delta_{\delta(R \mid Q)} .
$$

\section{3 局部操作与量子关联}

已有研究显示某些局部操作可以增加量子关 联 $[17,18]$. 在这一节, 结合间接测量模型, 我们讨论局 部操作与量子关联变化之间的关系. 在测量模型中, 局部操作可以看作是在系统 $Q$ 上的量子测量 $\Phi$. 由 上一节可知, 系统 $R Q$ 在局部操作前后的量子关联的 变化量可以由局部操作导致的系统 $Q$ 熵的增量、摘 交换以及系统 $R E$ 之间的纠缠给出, 即

$$
\Delta_{\delta(R \mid Q)}=\Delta S_{Q}-S\left(\rho^{Q}, \Phi\right)+E\left(\tilde{\rho}^{R E}\right) .
$$

它等价于 $\Delta S_{Q}-\Delta_{\delta(R \mid Q)}=S\left(\rho^{Q}, \Phi\right)-E\left(\tilde{\rho}^{R E}\right)$. 因为形 成纠缠熵不大于子系统的熵, 即 $S\left(\rho^{Q}, \Phi\right) \geqslant E\left(\tilde{\rho}^{R E}\right)$, 因此得到

$$
\Delta_{\delta(R \mid Q)} \leqslant \Delta S_{Q} .
$$

该不等式说明: 局部操作诱发的量子关联的增量不 超过在该系统上局部操作产生的熵增量. 由于局部 操作完全可能是熵减小的, 且量子关联有可能是不 增的, 即有 $\Delta_{\delta(R \mid Q)} \leqslant 0 \leqslant \Delta S_{Q}$. 该不等式等价于

$$
\delta(R \mid Q) \leqslant S\left(\rho^{Q}\right) \leqslant S\left(\Phi\left(\rho^{Q}\right)\right) .
$$

该不等式说明: 当两体复合系统初始启动于纯态时, 熵不减的局部操作一定不会导致的量子关联的增加.

同理, 系统 $Q E$ 在局部操作前后的量子关联的变 化量可以由局部操作导致的系统 $Q$ 熵的增量以及系 统 $R E$ 之间的纠缠给出, 即

$$
\Delta_{\delta(E \mid Q)}=\Delta S_{Q}+E\left(\tilde{\rho}^{R E}\right) .
$$

由于系统 $R E$ 之间的纠缠是非负的, 结合式 (29) 得到

$$
\Delta_{\delta(R \mid Q)} \leqslant \Delta S_{Q} \leqslant \Delta_{\delta(E \mid Q)} .
$$

该不等式说明: 局部测量产生的对应系统上的熵增 量不小于该测量诱发的与之关联的系统之间的量子 关联的增量, 也不大于与测量装置之间产生的量子 关联.

由于局部操作完全可能是熵减小的, 因此不等 式 (30) 的右边等式可以写成

$$
\Delta S_{Q} \leqslant 0 \leqslant \Delta_{\delta(E \mid Q)} .
$$

该不等式说明: 当两体复合系统初始启动于积状态 时, 熵不增的局部操作完全可以导致的量子关联的 增加.

\section{4 结论}

我们结合间接测量模型, 讨论了辅助量子系统、 被测量子系统以及测量装置之间关联分布, 研究结 果显示被测系统上的测量诱发的辅助系统和被测系 统之间的量子关联的减少量与被测系统和测量装置 之间的量子关联的增加量的和等于熵交换, 正好等 于测量诱发的全部关联的一半. 我们也研究了局部 操作与量子失协之间的关系, 研究结果表明局部测 量产生的熵增量不小于该测量诱发的辅助系统和被 测系统之间量子关联的增量, 不大于被测系统和测 量装置之间产生的量子关联. 我们的结果有助于理 解量子计算机中存储单元、量子计算机其他部分以 及带来噪声的环境之间关系, 更好地理解它们之间 熵的耗散与 Landauer 原理之间的关系. 


\title{
参考文献
}

1 Ozawa M. Uncertainty relations for noise and disturbance in generalized quantum measurements. Ann Phys, 2004, 311(2): 350-416

2 Buscha P, Heinonenb T, Lahtib P. Heisenberg's uncertainty principle. Phys Rep, 2007, 452: 155-176

3 Maccone L. Entropic information-disturbance tradeoff. Europhys Lett, 2007, 77: 40002

4 Buscemi F, Hayashi M, Horodecki M. Global information balance in quantum measurements. Phys Rev Lett, 2008, $100: 210504$

5 Luo S L. Information conservation and entropy change in quantum measurements. Phys Rev A, 2010, 82: 052103

6 Luo S L, Li N. Decoherence and measurement-induced correlations. Phys Rev A, 2011, 84: 052309

7 Cheong Y W, Lee S W. Balance between information gain and reversibility in Weak Measurement. Phys Rev Lett, 2008, 109: 150402

Schumacher B. Sending entanglement through noisy quantum channels. Phys Rev A, 1996, 54: 2614-2628

Henderson L, Vedral V. Classical, quantum and total correlations. J Phys A-Math Gen, 2001, 34: 6899

10 Ollivier H, Zurek W H. Quantum discord: A measure of the quantumness of correlations. Phys Rev Lett, 2001, 88: 017901

11 Modi K, Brodutch A, Cable H, et al. The classical-quantum boundary for correlations: Discord and related measures. Rev Mod Phys, 2012, 84: 1655-1707

12 Nielsen M A, Chuang I L. Quantum Computation and Quantum Information. Cambridge: Cambridge University Press, 2000

13 Holevo A S. Statistical problems in quantum physics. Probl Inf Transm, 1973, 330: 104-119

14 Roga W, Fannes M, Życzkowski K. Universal bounds for the Holevo quantity, coherent information, and the Jensen-Shannon divergence. Phys Rev Lett, 2010, 105: 040505

15 Koashi M, Winter A. Monogamy of quantum entanglement and other correlations. Phys Rev A, 2004, 69: 022309

16 Xi Z J, Lu X M, Wang X G, et al. Necessary and sufficient condition for saturating the upper bound of quantum discord. Phys Rev A, 2012, 85: 032109

17 Streltsov A, Kampermann H, Bruß D. Behavior of quantum correlations under local noise. Phys Rev Lett, 2011, 107: 170502

18 Hu X Y, Fan H, Zhou D L, et al. Necessary and sufficient conditions for local creation of quantum correlation. Phys Rev A, 2012, 85: 032102

\section{Measurement-induced correlation distribution}

\author{
XI ZhengJun* \& LI YongMing \\ College of Computer Science, Shaanxi Normal University, Xi'an 710062, China
}

\begin{abstract}
It is well known that the disturbance induced by measurement can cause the change of the correlations in the quantum system with the auxiliary system and the apparatus. In this paper, using entropy change and quantum discord, we first discuss measurement-induced correlation distribution and derive a tradeoff for total correlation and quantum correlation in the indirect measurement model. In this basis, the relation between the behavior of quantum correlation under local operation and the correlation distribution is established.
\end{abstract}

quantum measurement, quantum correlation, entropy exchange, entropy increase

PACS: 03.67.-a, 03.67.Pp, 03.65.Yz

doi: $10.1360 / 132012-903$ 